\title{
Image processing of weld seam for Al alloy pulsed GTAW based on
}

\section{vision sensing}

\author{
Yunxia Chen ${ }^{1,2, a^{*}}$, Su Zhao ${ }^{1, b}$, XiaoJing Wang ${ }^{3, c^{*}}$
}

1. School of Mechanical and Electrical Engineering, Shanghai Dianji University, ShangHai, China

2. Shanghai Jiao Tong University, Shanghai 200240, China

3. Jiangsu University of science and technology, ZhenJiang 212003, China

acyx1978@yeah.net, bhaosu@sdju.edu.cn, xjwang.0@163.com

Keywords: arc welding robot, vision sensing,image processing,seam tracking

Abstract. In recent years, machine vision sensors have been widely used to track weld seam. A suitable way is designed to process image of aluminum alloy pulsed gas tungsten arc welding process experiments in this work. With the passive vision sensor to capture some ideal images, we adopt and compare different image processing methods to extract useful information, including target location, double filter, edge detection and seam centerline extraction. It is shown that the method has good effect on seam centerline extraction and seam tracking.

\section{Introduction}

Recently, the development of robotic arc welding systems has progressed rapidly as a result of the continuing demand for improved methods of manufacturing industrial products. Especially, aluminum alloy pulsed gas tungsten arc welding (GTAW) is widely used in the aerospace, automotive and other industries. However, the robotization of the arc welding process is often influenced by errors of manufacturing and assembling, distortion. It is urgent for seam tracking during the arc welding process to detect the weld position accurately ${ }^{[1-3]}$.

In engineering practice, visual sensors such as charged-coupled device (CCD) cameras are widely used to detect the weld position, and many seam tracking systems utilizing those vision techniques can achieve accuracies suitable for the arc welding control process ${ }^{[4-8]}$. The methods presented above greatly relied on weld images captured from a CCD camera used the gradient information of the weld. However, in actual welding processes clear and contrasting weld images are seldom obtained. Optical noise such as arc glares, welding spatters and smoke which affects the weld images severely and makes it difficult to obtain the clear weld images and exact weld edge or position information.

In this study an effective seam image processing algorithm to extract the seam centerline is developed with a passive vision sensor which is used for measuring the detailed geometric features of the weld joint to be welded, and then achieving robot path correction from the measured results.

\section{Experimental system}

The experiment system is shown in Fig. 1. The system mainly includes a robot, a weld power supply, a vision sensor and a host computer. The robot is a six-degree industry robot made by YASKAWA Corporation. The weld power supply consists of an OTC AVP500 inverter and an 
HC-71 feeder. The vision sensor device is composed of a CCD camera which is in charge of capturing the real-time images for seam information extraction so that the host computer can calculate deviation to help the robot planning the welding path and complete the welding task. The weld pool image was obtained by the visual sensor used a filter system to filter the intense arc light.

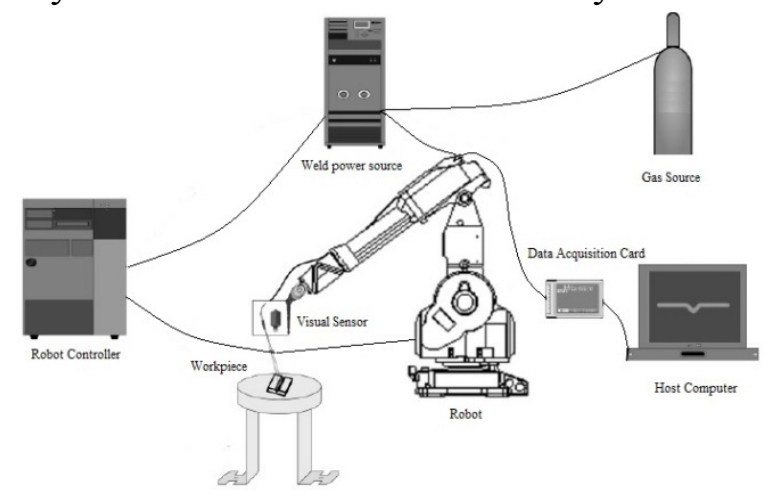

Fig.1 The schematic diagram of robotic GTAW system

The base material is AL alloy with dimensions of $300 * 100 * 3 \mathrm{~mm}$, GTAW welding parameters are as following: the base current is $30 \mathrm{~A}$ and peak current is $230 \mathrm{~A}$, pulse frequency is $2 \mathrm{HZ}$, duty cycle of pulse duration is 50 percent, tungsten anode diameter is $3.2 \mathrm{~mm}$, feed speed is $10 \mathrm{~mm}$ per second, welding speed is $3 \mathrm{~mm}$ per second, shielding gas is 99.99 percent, Ar argon flow is $15 \mathrm{~L}$ per minute.

\section{Image processing}

During the Al alloy pulsed GTAW, the image processing algorithms should have good adaptabilities that ensure to reliably extract the geometry characteristic parameters of seam and pool under various kinds of conditions. An image containing the two objects in position for the butt-weld operation must first be captured. This image must view the entire seam. Appropriate image processing is performed to allow for the boundary detection of the two objects to take place. The image of welding seam and molten pool with dimensions of $768 * 576$ Pixel taken by the CCD camera is shown in Fig.2(a). The images are captured on the moment of base pulse current. From Fig.2 (a), the weld seam and pool can be seen clearly, which is beneficial to extract the edges of seam and pool. The acquisition of image will directly affect the control of welding process on precision and forming quality. In the seam tracking of welding robot with passive vision, the purpose of image processing is to extract the geometry characteristic parameters of the seam and pool quickly and accurately. The accuracy and timeliness of image processing will have a decisive impact on the seam tracking control and forming quality, so the image processing is very important.

In order to reduce the redundant information and accelerate image processing speed, a region of interest (ROI) with dimensions of $355^{*} 139$ Pixel in front of the molten pool is selected. ROI image is shown in Fig.2 (b).In the ROI image, seam can be obtained quickly. 


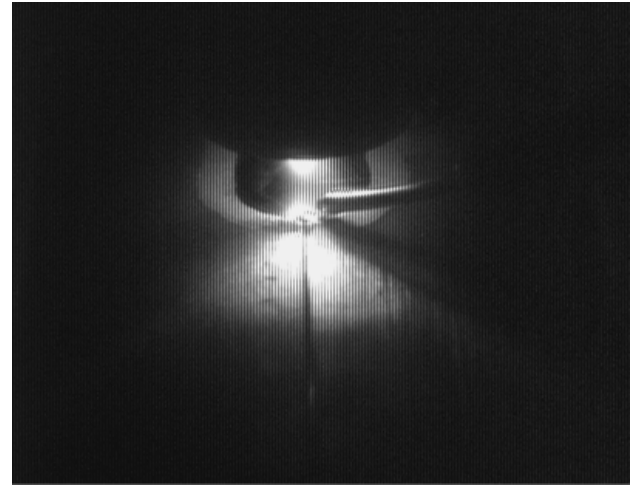

(a) The welding image

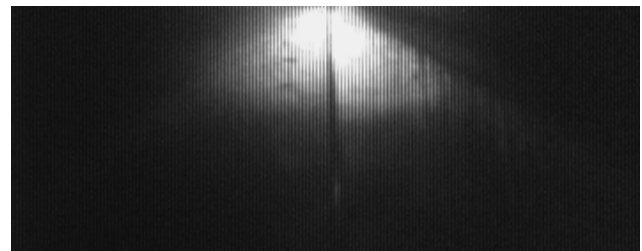

(b) ROI selecting

Fig. 2 The welding image during the GTAW process.

The average gray value of ROI image is nearly 240 , while weld seam gray values range from 25 to 255. At the same time, the whole image has obvious vertical lines interference, mainly due to droplet transfer which can make weld arc cyclically flash and form coherent lights with the same frequency. Under those coherent lights it is inevitable for images captured from the CCD camera with bright and dark stripes. The gray value of white stripes is 29 higher than that of black stripes always. Binarization algorithms is not effective because the weld seam images have not uniform regions with good separation between the weld and its surroundings of weldments.

To obtain clear and contrast weld images, a method for weld seam detection is introduced. This method uses a single image captured by a robot mounted camera referred to as the eye-in-hand configuration. The overall method contains 4 steps: double filter, seam edge detection; Weld seam center line fitting and extraction. Each step is discussed in detail in the following subsections.

\section{Double filter}

As is known to all, GTAW welding process is a complex and variable process, such as the welding splash, dust and metal reflecting light which often make the image sensor contains a lot of noise. In order to avoid the noise interference in the subsequent image processing, it is necessary to remove the noise in the original image. According to the property of the original image features and image noise, test by Prewitt, Sobel, Log, Laplace and Gauss on the weld image processing, image after filtering is shown in Figure 3 (a) (f). Compared with Figure 3 (a) (d), the weld pool and seam can be clearly seen in Figure 3 (e), however the vertical lines interference still exists.

Median filter is used to remove random noise and to maintain image sharpness. The method of obtaining the median value is as follows: let each point in the $5 * 5$ pixel range be arranged according to its numerical value. The median value may then be obtained. If the number of points within the mask is an even number, then the median value is the average of the two numerical values. Figure 3 (f) shows the vision processing result by Gauss \& Median double filter.

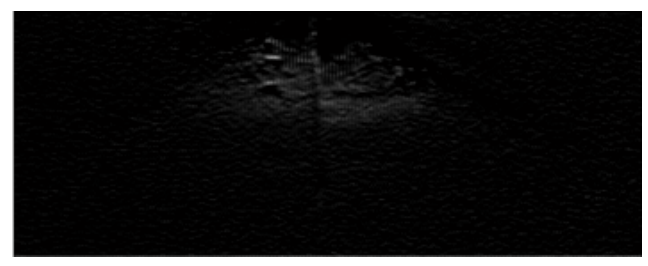

(a) Prewitt filter

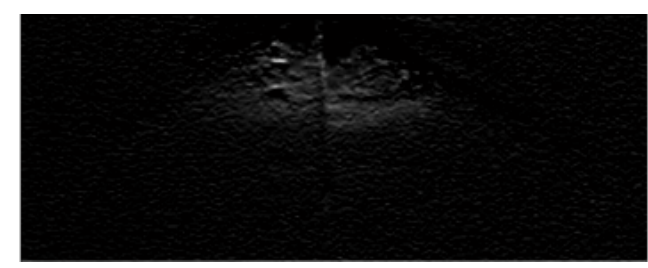

(b) Sobel filter 


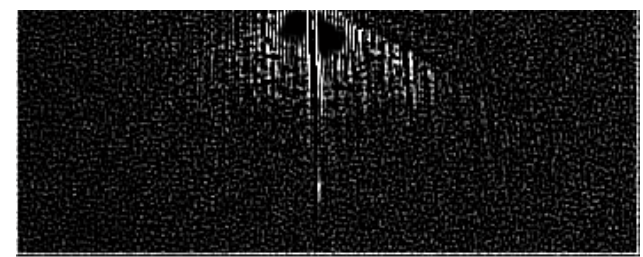

(c) Log filter

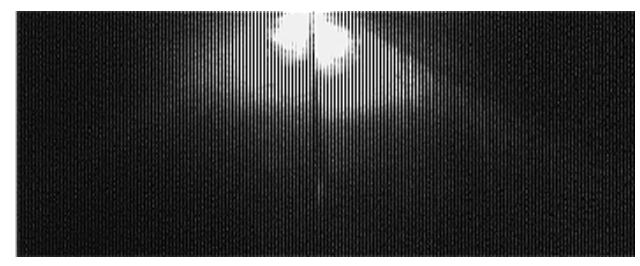

(e) Gauss filter

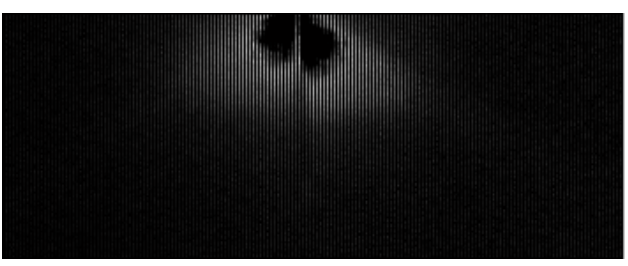

(d) Laplace filter

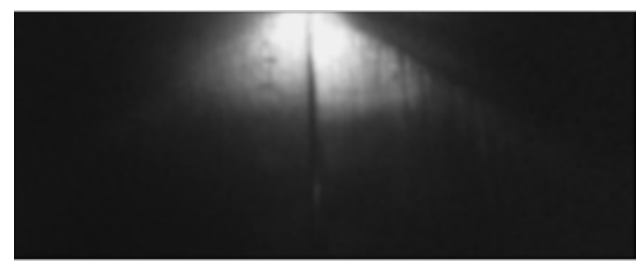

(f) Gauss \& median filter

Fig.3 Compare of some typical smoothing algorithms.

\section{Seam edge detection}

Now that we have acquired filtered data, we find the reference points for path planning of the weaving motion. The Roberts, Canny, Prewitt and Log edge identification algorithms are used in this paper. The resulting edge images are shown in Fig. 4(a) (e). The grayscale edge image produced by each algorithm is converted to a black and white binary image using an adaptive threshold. To ensure that the edges are unbroken and are clearly defined lines, the binary image is dilated as shown in Fig. 4 (f).

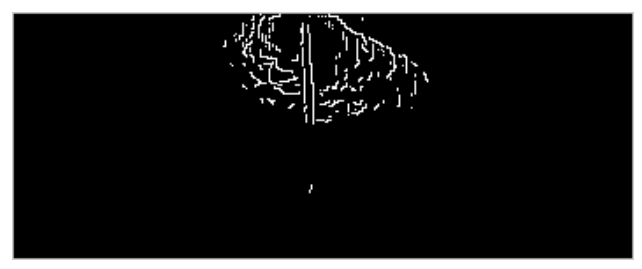

(a) Roberts

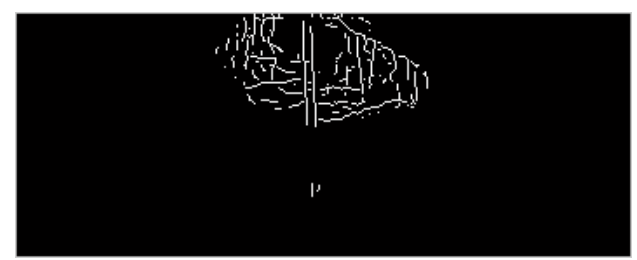

(c) Prewitt

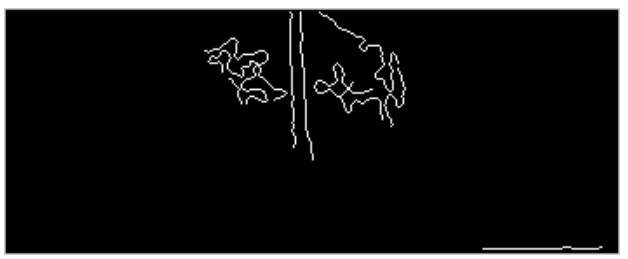

(e) Automatic thresholding

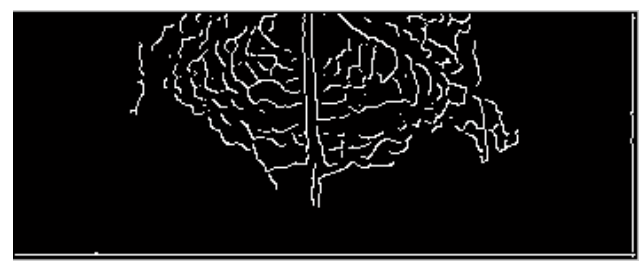

(b) Canny

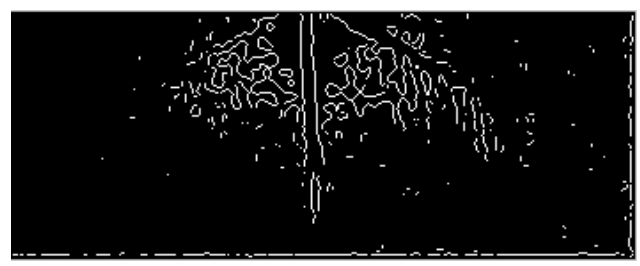

(d) $\log$

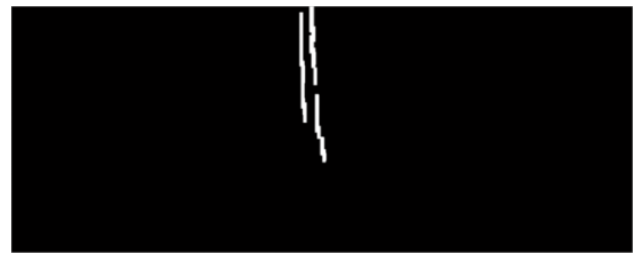

(f) Dilation

Fig.4 Compare of edge scan

\section{Weld seam center line fitting and extraction}

The final stage of pre-processing is performing edge identification on the dilation image, which 
should be eroded so that the edge lines have a width of one pixel. In order to guarantee the welding torch to track along the seam precisely, the accurate seam centerline position must be obtained. Here the following method is taken:

(1) To scan the eroded image (Fig.5 (a)) from left to right to find the boundary point whose pixel value is equal to 255 . If two points are found, then the scan is stopped at once. The first point is the left boundary point $P\left(i_{1}, j\right)$, the other point is the right boundary point $P\left(i_{2}, j\right)$. The variable $i, j$ which is initialized to 0 is increased with 1 automatically if the top and bottom points are found on each scan.

(2) If only one point is found, according to its distance to judge which boundary point, then the other boundary point will be calculated by linear interpolation.

(3) If no point is found, $P\left(i_{1}, j\right)$ and $P\left(i_{2}, j\right)$ can be calculated by linear interpolation.

Based on the image processing, the seam center line equation $x=\left(i_{1}+i_{2}\right) / 2$ (that is the center point horizontal coordinate) can be obtained, and the deviation $d(t)=x_{0}-x$ from the pool center point to the seam centerline in the present moment can be calculated in real-time, the result line is got by connecting all center points as shown in Fig.5(b).

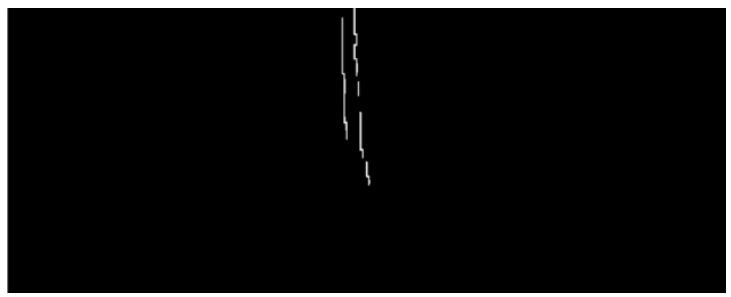

(a) The erosion image

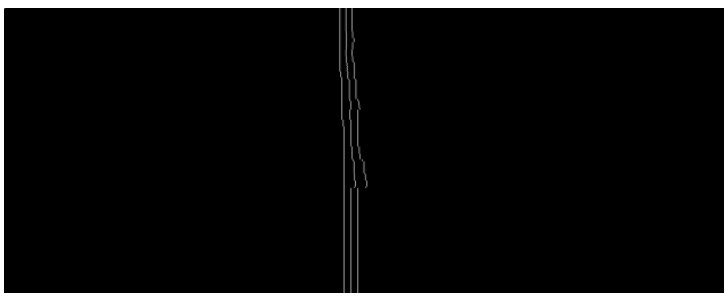

(b) Fitted image

Fig.5 Seam center fitting

\section{Conclusions}

In this paper a flexible seam detection method has been introduced. The clear welding image was acquired by using a self-designed passive vision sensor, which is the prerequisite for seam tracking during robotic welding process. Through careful analysis of the welding images, an improved double filter algorithm of image processing was proposed, and the seam center line position was accurately extracted. The next task will be to analyze the deviation accurately and send the deviation to an executing agency to achieve quantitative regulation through a large amount of experiments and research.

\section{Acknowledgements}

I would like to express my gratitude to Dr Yanling $\mathrm{Xu}$ for assistance with the experiments. This work is supported by Shanghai Key Laboratory of Materials Laser Processing and Modification.

\section{References:}

[1] Jae Seon Kim,Young Tak Son,Hyung Suck Cho,Kwang II Koh, A robust visual seam tracking system for robotic arc welding, J. Mechatronics. 6(2) (1996) 141-63.

[2] XuY.L,Zhong J.Y,Ding M.Y,Chen H.B,Chen S.B. The acquisition and processing of real-time information for height tracking of robotic GTAW process by arc sensor. Int J Adv Manuf 
Technol. 65 (2013) 1031-43.

[3] Jeong S.K,Lee G.Y,Lee W.K,Kim S.B. Development of high speed rotating arc sensor and seam tracking controller for welding robots. Ind Electron. 2 (2001) 845-50.

[4] Shi YH,YooWS,NaSJ. Mathematical modeling of rotational arc sensor in GMAW and its applications to seam tracking and end point detection. Sci TechnolWeldJoining. 11 (2006) 723-30.

[5] Xu P.Q.,Xu G.X., Tang X.H., Yao Sh., A visual seam tracking system for robotic arc welding. The International Journal of Advanced Manufacturing Technology. 37 (2008) 70-5.

[6] ZhangW.Z,Chen Q.,Zhang G.X.,Sun Zh.G.,Du D.,Seam tracking of articulated robot for laser welding based on visual feedback control, RoboticWelding,Intelligence and Automation.362 (2007) 281-7.

[7] Liang Y.L.,Ling J.F.,Xin Zh.,Xiang L., Image processing of seam tracking system using laser vision. RoboticWelding, Intelligence and Automation, 362 (2007) 319-24.

[8] Chang-HyunKim,Tae-YongChoi,Ju-JangLee,JeongSuh,Kyoung-TaikPark, Hee-Shin Ka. Development of welding profile sensor and its application, In: Proceedings of the International conference on smart manufacturing application KINTEX,Gyeonggi-do,Korea. (2008) 24-9.

[9] BaeKY,LeeTH,AhnKC. An optical sensing system for seam tracking and weld pool controlling as metal arc welding of steel pipe. J MATER PROCESS TECH. 120 (2002) 458-65. 